\title{
Fachlich-biographische Notizen zur publizistischen Tätigkeit katholischer Geistlicher in der deutschen Presse des 19. Jahrhunderts
}

\section{Folge}

In der Literatur zur Kulturkampfzeit begegnet dem Leser gelegentlich - als ferne Reminiszenz - der Begriff "Kaplanspresse“. Die von Zeitgenossen offenbar in abschätzigem Sinne verwendete Bezeichnung spielt auf die Tatsache an, daß nicht wenige katholische Zeitschriften und Zeitungen Deutschlands, die gerade während und wegen des Kulturkampfes an Zahl erheblich zunahmen, von Geistlichen geleitet oder doch mitgestaltet, vielfach auch gegründet wurden. Besonders die kleineren Blätter unter den Neugründungen seien, so schreibt verallgemeinernd Kisky in der 50-Jahr-Festschrift des Augustinus-Vereins ${ }^{1}$, „fast durchweg von Geistlichen redigiert" worden. Aber weder die ökonomische Motivierung, die Kisky formuliert: die im Kulturkampf amtsenthobenen Geistlichen seien um des Broterwerbs willen auf Arbeit bei den Zeitungen angewiesen gewesen ${ }^{2}$, - noch eine idealistische Deutung Paul Weilbächers, wonach die durch staatlichen Eingriff gewissermaßen arbeitslos gewordenen Seelsorgepriester jetzt "ein neues Arbeitsfeld“ gesucht und gefunden hätten ${ }^{3}$, reichen zur vollständigen Erklärung der "Kaplanspresse“ aus. Tatsache ist vielmehr, daß die katholische Presse Deutschlands schon vor dem Kulturkampf in ihren Redaktionen mit Geistlichen reich bestückt war und daß sich Geistliche seit den Anfängen der katholischen Presse in Deutschland auch als Gründer und zuweilen selbst als Verleger mit auffällig hohem Zahlenanteil beteiligt haben. ${ }^{4}$ Eine verhältnismäßig einfache Erklärung findet diese Erscheinung darin, daß der katholische Bevölkerungsteil überhaupt nur in einer akademischen Fachrichtung Gebildete nicht nur in ausreichender Zahl hervorbrachte, sondern auch freizusetzen vermochte, nämlich in der Theologie. Wurden für ein Preßunternehmen, wie es in der Sprache der Zeit heißt, literarisch gebildete und befähigte Kräfte gesucht, - von journalistischer Ausbildung im engeren Sinne konnte noch keine Rede sein, - so kamen neben einigen wenigen Juristen und Oberlehrern eigentlich nur Theologen dafür infrage. Sie brachten zudem gewissermaßen von Natur aus den Vorzug mit, daß unter ihrer Obhut die Rechtgläubigkeit katholischer Publizistik gesichert schien.

In zahlreichen Darstellungen der katholischen Bewegung Deutschlands und auch in den Spezialarbeiten über die entsprechende Pressegeschichte stößt man also immer wieder auf Spuren dieser starken geistlichen Beteiligung. Einen genauen und geordneten Uberblick gibt es nirgends. Er ist jedoch wichtig, will man verallgemeinernden Urteilen vorbeugen, und er könnte einen Beitrag zur Sozialgeschichte der katholischen Presse und damit allgemein der deutschen Presse im 19. Jahrhundert liefern. 
Im Rahmen eines Seminars am Institut für Publizistik der Universität Münster, das dem Thema „Weltanschauung und Publizistik " gewidmet war, leiteten die zahlreichen bei der Behandlung des Aspektes Katholizismus und Publizistik aufgefundenen Namen geistlicher Gründer, Herausgeber und Redakteure zu einer Päzisierung der Frage nach der tatsächlichen Beteiligung dieses Personenkreises an der katholischen Presse. $\mathrm{Da}$ in der Publizistik-Geschichte ganz allgemein biographische Probleme, von größeren Monographien abgesehen, eher zu den vernachlässigten Gebieten gehören, rechtfertigte die hier aufgekommene Fragestellung eine ausführliche, über das übliche Ausmaß von Seminarstudien hinausgehende Behandlung. Es galt herauszufinden und zusammenzutragen: 1. die Namen und Daten möglichst vieler katholischer Geistlicher, die im Gebiet des nachmaligen Deutschen Reiches in der katholischen Presse tätig waren, und 2. möglichst zuverlässige Angaben über die Art des Verhältnisses, in welchem die zu beschreibenden Personen zu Presseorganen standen. Die Ergebnisse sollten möglichst knapp in lexikonartigen Stichworten zusammengefaßt werden, unter Hinzufügung von Hinweisen auf die Fundstellen, aus denen die Sach- und Personalangaben geschöpft wurden. Entstanden sind auf diese Weise biographisch-fachliche Kurzartikel, wobei die Betonung auf fachlich liegt: Die Berührungspunkte der jeweiligen Person mit der Publizistik, ihre Leistungen für Zeitungen oder Zeitschriften, waren das in erster Linie Erwähnenswerte; die wichtigsten Lebensdaten wurden hinzugefügt, soweit dies ohne Heranziehung von speziellen Nachschlagmitteln, etwa Personalschematismen, möglich war. Die hier in der Folge veröffentlichten Personaldaten wollen und können also nicht das Nachschlagen in den jeweiligen Schematismen ersetzen.

Die publizistikbezogenen und also hier wiedergegebenen Informationen sind aus recht verschiedenen, im allgemeinen jedoch (aus arbeitsökonomischen Gründen) aus sekundären Quellen gewonnen. Dies brachte für den Bearbeiter und bringt für den Benutzer ein gewisses Risiko mit sich: Längst nicht alle Darstellungen, aus denen geschöpft wurde, sind im nötigen Maße zuverlässig. Selbst ein Standardwerk wie Karl Bachems Geschichte der katholischen Presse ist im Biographischen nicht mit der nötigen Sorgfalt geschrieben, - andere Spezialuntersuchungen bieten, auf Grund ihrer besonderen Fragestellung, keine voll befriedigenden Auskünfte. Selbst ein biographisches Nachschlagewerk wie das „Katholische Deutschland“ von Kosch ist, wie vergleichende Beobachtungen ergeben haben, nicht in jeder Hinsicht zuverlässig. Im allgemeinen wurde, soweit möglich, der kontrollierende Vergleich als Korrekturverfahren herangezogen; in vielen Fällen fehlte es jedoch einfach an Vergleichsdaten. Das an sich gebotene Verfahren: die Gewinnung der Daten aus primären Quellen, d. h. aus Akten oder wenigstens aus den in Frage kommenden publizistischen Originalen (Zeitungen und Zeitschriften) selbst, war nur in einigen Ausnahmefällen anwendbar. Der allein mit dem Ausfindigmachen und Aufsuchen der publizistischen Primärquellen verbundene Aufwand an Zeit, materiellen Mitteln und Arbeitskraft hätte in keinem vernünftigen Verhältnis zur ziemlich begrenzten Aufgabenstellung (Schaffung eines Überblicks) gestanden. Die Artikelchen sind also verbesserungsfähig und in vielen Fällen -bedürftig, - weshalb sie auch in der Überschrift nur als Notizen deklariert sind, - und sie spekulieren durchaus auf die Mitarbeit kundiger Leser.

Auf eine besondere Fehlerquelle muß noch aufmerksam gemacht werden: Bei einigen wenigen Personen ist zwar gut begründeter Verdacht, aber nicht letzte Sicherheit darüber gewonnen worden, ob es sich um Geistliche oder Laien handelt. Die entsprechenden Namen sind mit einem eingeklammerten Fragezeichen (?) gekennzeichnet. 
Die Groß-Ordnung der ganzen hier in Fortsetzung zu veröffentlichenden Reihe ist die alphabetische Reihenfolge der Namen. Die innere Ordnung der Einzel-Artikel ist folgende: Nach dem Namen, der (nicht immer genau formulierbaren) publizistischen Tätigkeits- oder Berufsbezeichnung und der Zusammenfassung der wichtigsten publizistischen und Lebensdaten wird die Fundstelle(n) der Informationen in Form von Kennziffern oder -buchstaben angeführt; dieser Chiffre folgt nach einem Komma die angezogene Seitenzahl, - falls erforderlich, ist zwischen Chiffre und Seitenzahl noch die Bandbezeichnung (bei mehrbändigen Werken) eingeschoben. Die Aufschlüsselung der Kennziffern und -buchstaben - und damit den Kern der ausgewerteten Literatur - findet der Leser in diesem Heft auf der dritten Umschlagseite. Gibt es zu einzelnen Personen außer den in der Zusammenfassung gegebenen Fakten noch bemerkenswerte zusätzliche Einzelheiten anzuführen, die das Bild ergänzen, aber auf andere Fundstellen zurückgehen, so folgt nach der ersten chiffrierten Angabe der Hauptfundstelle(n) ein Gedankenstrich, danach die Mitteilung des ergänzenden Sachverhalts, der wiederum abschließend mit Kennziffer und Seitenzahl belegt wird.

Die Nennung der jeweiligen Fundstellen bewirkt zweierlei: Sie gleicht den Nachteil, der durch Auswertung von Sekundärquellen im Hinblick auf die Zuverlässigkeit gegeben ist, aus, indem sie den Benutzer ggf. schnell zur Fundstelle zurückführt und ihm so eigenes Urteil gewinnen hilft, und sie ist ferner ein allgemein weiterführender Literaturhinweis in nuce.

Aus der erstaunlichen Reihe teils mehr oder minder bekannter, teils heute wieder ganz vergessener Namen sind schließlich auch über die publizistisch und sozialgeschichtlich interessante Information hinaus einige Denkanstöße zu gewinnen, deren Richtung den nationalen Rahmen unserer Sammlung sprengt: Zwar sind die aus den einzelnen Artikelchen sich summierenden publizistischen Leistungen innerhalb einer inzwischen historisch gewordenen Zeit im Rahmen einer ganz bestimmten, von genau beschreibbaren politischen und philosophischen Ideen beherrschten $\mathrm{Ge}$ sellschaftsordnung vollzogen worden: Geistliche, zunächst in ihrer Eigenschaft als akademisch Gebildete und als „Volkslehrer", wie sie vom Priester-Journalisten Kolping treffend bezeichnend worden sind, haben als fachlich nicht vorgebildete Amateur-Publizisten einem publizistisch rückständigen Volksteil zur Verbesserung seiner Lage verholfen, jedoch, wie man nachträglich feststellen kann, mit einem unverhältnismäßig hohen Kräfteverschleiß. Fachjournalisten mit einer $z$ weckentsprechenden Spezialausbildung hätten, wenn man sie nur in ausreichender Zahl zur Verfügung gehabt hätte, bei geringerem Aufwand größeren Effekt erzielt. In ähnlicher Situation befinden sich heute nicht wenige, von christlichem Einfluß mitbestimmte Gesellschaftsordnungen in Entwicklungsländern. Auch für sie scheint die Rolle des publizistischen Volkslehrers von christlicher Seite noch nicht hinlänglich durchdacht worden zu sein.

\section{Anmerkungen:}

1. Wilhelm Kisky: Der Augustinus-Verein zur Pflege der katholischen Presse von 1878 bis 1928, Düsseldorf 1928, S. 59.

2. ebenda.

3. Paul Weilbächer (Hrsg.): Augustinus-Verein zur Pflege der katholischen Presse. Festgabe zum silbernen Jubiläum. Gefeiert zu Cöln am 23. August 1903, Krefeld 1903, S. 7.

4. Vgl. etwa Rudolf Pesch: Die kirchlich-politische Presse der Katholiken in der Rheinprovinz vor 1848, Mainz 1966, oder Michael Schmolke: Adolph Kolping als Publizist, Münster 1966. 
Abel, Wilhelm, SVD, geboren 4. 12.1853 in Roßbach bei Fulda, gest. 28. 10. 1909 in Steyl, besuchte die Schule in Fulda und trat dann ins Collegium Germanicum in Rom ein. Nach Promotion zum Dr. phil. wegen Krankheit nicht zum Priester geweiht, wurde er Lehrer in Steyl, 1881-82 Lehrer bei den Dominikanern in Venlo und 1885 bei den Benediktinern in St. Ottilien. 1886 kehrte er nach Steyl zurück, wo er 1888 zum Priester geweiht wurde. Seit 1886 Mitarbeiter der Stadt Gottes, seit 1889 Herausgeber und Schriftleiter derselben und dazu des St. Michaels-Kalenders, beide Zeitschriften leitete er bis $\mathrm{zu}$ seinem Tode. (Hermann Fischer, Arnold Janssen, Steyl 1919, S. 200).

Aberle, Moritz von, Redakteur, geb. 25. 4. $1817 \mathrm{zu}$ Rottum bei Biberach, gest. 3. 11. $1875 \mathrm{zu}$ Tübingen, studierte in Tübingen, wurde 1842 Priester, 1843 Repetent am Wilhelmsstift, 1845 Gymnasialprofessor und wirkte 1850-75 als Professor für neutestamentliche Exegese und Moraltheologie. Hauptmitarbeiter der Tübinger Theologischen Quartalschrift. KK, 3.

Abs, Josef, Herausgeber, geb. 20. 5. 1889 zu Oespel, ging als Missionar nach Indien, wonach er Indologie an der Universität Bonn betrieb und Privatgelehrter wurde. Herausgeber der Halle der Religionen. KK, 6 .

Abt, Anton, Pseudonym Walther von Münich, Chefredakteur, geb. 26. 12. 1841 zu Seelenberg in Nassau, gest. 16. 2. 1895 zu Limburg an der Lahn, studierte in Mainz, widmete sich seit 1865 der Seelsorge, wurde 1866 Subregens in Montabaur, 1867 auch Gymnasiallehrer daselbst, übernahm 1882 eine Kaplanstelle in Frankfurt a. M., war seit 1884 Pfarrer in Königstein, seit 1885 Domkapitular und Stadtpfarrer in Limburg. Zeitweilig Redakteur des Anzeigers für die katholische Geistlichkeit Deutschlands. Vorwiegend Erzähler. KK, 7. - Chefredakteur des Anzeigers für die katholische Geistlichkeit Deutschlands ist (1900) Professor Ludwig Abt. KU, 76.

Adhterfeld, Johann Heinrich, Mitbegründer und Herausgeber, geb. 1. 6. 1788 zu Wesel, gest. 11. 5. $1877 \mathrm{zu}$ Bonn, studierte in Köln und Münster, wirkte dann in der
Seelsorge, wurde 1817 Professor der Theologie in Braunsberg, 1826 in Bonn, 1843 in die hermesianischen Streitigkeiten verwickelt und seiner Lehrtätigkeit enthoben, die ihm erst zwei Jahrzehnte später wieder zugestanden wurde. Seit 1832 Herausgeber der Zeitschrift für Philologie und katholische Theologie, des Hauptorgans der Hermesianer. KK, 9. - Die Zeitschrift für Philologie und katholische Theologie wurde von Braun und Achterfeld in Bonn herausgegeben. 13,14. - Die hermesianische Zeitschrift für Philologie und katholische Theologie wurde $1832-53$ von den Bonner Professoren Achterfeld, Braun, v. Droste, Scholz und Vogelsang herausgegeben. 1, I, 244 vgl. 9, 20.

Ah, Joseph Ignaz von (Pseudonym Hartmann von Baldegg), Gründer, geb. 15. 12. $1834 \mathrm{zu}$ Sachseln in Obwalden, gest. 1. 9. 1896 zu Kerns, Bauernsohn, im Stift Einsiedeln ausgebildet, studierte am Priesterseminar in Chur, widmete sich seit $1857 \mathrm{der}$ Seelsorge in Bern, Freiburg im Uchtland und Sterns, wo er das Nidwaldener Volksblatt gründete und wofür er über 1500 in der ganzen Schweiz beachtete Wochenberichte schrieb. Seit 1867 Pfarrer von Kerns in Obwalden, trat auch als Dichter, Prediger und Historiker hervor. KK 17.

Ahrenhold, Johann Wilhelm, Redakteur und Mitbegründer, geb. 1845 in Amöneburg, gest. 13. 5. 1921, wurde 1869 Priester, Kaplan in Fulda, Dr. theol. et. phil., Lehrer am Knabenseminar daselbst, 1895 Professor, 1897 Domkapitular, 1906 Generalvikar, 1907 apostolischer Protonotar, Mitbegründer der Fuldaer Zeitung. 6, 53. Chefredakteur der Fuldaer Zeitung. 19, 83.

Aichinger, Georg, Redakteur und Verleger, geb. 20. 4. $1835 \mathrm{zu} \mathrm{Kogl,} \mathrm{gest.} \mathrm{15.} \mathrm{2.} 1916$ zu Straubing, 1867-97 Redakteur des Straubinger Tagblattes, Priester im Bistum Regensburg, (Auskunft des Archivs des Bistums Regensburg). - Seit 1. 4. $1868 \mathrm{Be}$ sitzer des Tagblatt in Straubing. 18, 219 vgl. 9, 64 .

Alleker, Johann, Herausgeber, geb. 30. 12. 1817 zu Köln, gest. 20. 7. 1889 zu Neuenahr, zuerst in der Seelsorge, dann im Schul- 
dienst tätig, seit 1863 Seminardirektor in Brühl. Herausgeber der Katholischen Zeitschrift für Erziehung und Unterricht in Neuß bzw. Düsseldorf. KK, 28.

Allioli, Joseph von, Mitherausgeber und Mitarbeiter, geb. 10. 8. 1793 zu Sulzbach, gest. 22. 5. $1873 \mathrm{zu}$ Augsburg, studierte in Landshut, Dr. theol,, danach Studien in Wien, Rom und Paris, habilitierte sich 1821, 1823 Professor in Landshut, 1826 in München, 1830 Rektor der Universität daselbst, 1835 Domherr in Regensburg, 1850-53 bayrischer Landtagsabgeordneter. KK, 29. - Mitherausgeber des Repertorium für katholisches Leben, Wirken und Wissen. 13, 214. - Mitarbeiter der Neuen Sion. $13,224$.

Altum, Bernhard, Mitherausgeber, geb. 31. 12. $1824 \mathrm{zu}$ Münster i. W., gest. 1. 2. 1900 zu Eberswalde, studierte Theologie, Philosophie und Naturwissenschaften in Münster, 1849 Priesterweihe, wurde 1856 Reallehrer, 1858 Domvikar, 1859 Privatdozent für Zoologie in Münster und 1869 Professor an der Forstakademie in Eberswalde. Zoologe, Gegner Brehms. KK, 32. - Mitherausgeber der Zeitschrift Natur und Offenbarung. 9, 46.

Alzog, Johann Baptist, Herausgeber und Redakteur, geb. 29. 6. 1808 zu Ohlau, gest. 1. 3. 1878 zu Freiburg i. Br., wurde 1836 Professor am Klerikalseminar in Posen, 1844 Domherr, Professor und Regens in Hildesheim, 1853 Professor der Kirchengeschichte in Freiburg und beteiligte sich 1869 in Rom an den Vorarbeiten für das Vatikanum. KK, 33.- Gab seit 1857 in Freiburg das von ihm gegründete Freiburger Katholische Kirchenblatt heraus. 18, 43 vgl. 9, 46. - Die Theologische Monatschrift, 1850-51, wurde von ihm mitredigiert. 9, 46. - Dem Freiburger Katholischen Kirchenblatt wurden die in der erzbischöflichen Kanzlei redigierten Kirchlichen Anzeigenblätter und die früher von Alzog herausgegebenen Christlichen Kunstblätter beigelegt. 18, 44.

Amrhein, August, Herausgeber, geb. am 26. 8. $1847 \mathrm{zu}$ Weibersbrunn, studierte in Würzburg, promovierte daselbst zum Dr. phil., Dr. theol. und Dr. jur., wurde Pfar- rer in Eßfeld. Herausgeber der Franconia Sacra, vorwiegend Lokalhistoriker. KK, 37.

Anzenberger, Josef, Dr. (?), Herausgeber und ab 1842 auch Redakteur der Passauer katholischen Kirchenzeitung. 13, 214 f. Seit 1841 Redakteur der Blätter zur Belehrung und Erbauung für katholische Christen. 13, 207.

Arens, Bernhard, Redakteur, geb. 24. 7. $1873 \mathrm{zu}$ Hosingen in Luxemburg, Bauernsohn, wurde 1892 in Blyenbeek (Holland) Jesuit, später Gymnasiallehrer in Feldkirch (Voralberg), redigierte 1912-1916 und seit 1925 die Zeitschrift Katholische Missionen, schrieb daneben Erzählungen für Volk und Jugend. KK, 55.

Arenz, Carl, Publizist, schrieb religiöse Gedichte für das Rheinische Kirchenblatt (1845-49). 13, 220 u. 13, 44. - Mitarbeiter der Katholischen Blätter in Düsseldorf (Neuss, Köln, 1845-48). 13, 221.

Arndt, Augustin, Chefredakteur, geb. 22. 6. $1851 \mathrm{zu}$ Berlin, gest. 21. 7. $1925 \mathrm{zu}$ Bukarest, seit 1874 katholisch, wurde 1875 Jesuit, 1884 Theologieprofessor in Krakau, wo er bis 1895 wirkte, 1909 in Weidenau. 1894--1918 Schriftleiter des Katholischen Sonntagsblatt in Breslau. KK, 59.

Aschenbrenner, Beda, geb. 6. 3. 1756, gest. 24. 7. $1817 \mathrm{zu}$ Ingolstadt, Schüler des Aufklärers B. Werkmeister in Freising, wurde 1774 Benediktiner in Oberaltaich, 1786 Professor für Kirchenrecht und Geschichte daselbst, 1789 in Ingolstadt, wo er als erster Theologe seine Vorlesungen in deutscher Sprache abhielt, 1796 Abt seines Stiftes bis zu dessen Aufhebung, Publizist, mit staatskirchlichen Problemen beschäftigt, rationalistisch gefärbt. KK, 68 .

Aufhauser, Johann, Mitherausgeber, geb. 7. 9. $1881 \mathrm{zu}$ Moosham bei Regensburg, Bauernsohn, studierte in Innsbruck und München, erwarb den Grad eines Dr. phil. und Dr. theol., habilitierte sich 1911 für Kirchengeschichte in München, wirkte 1914 bis 1918 als Feldgeistlicher, wurde 1919 in Brüssel interniert, nachdem er inzwischen zum Professor für Missionswissenschaft ernannt worden war. Mitherausgeber der Zeitschrift für Missionswissenschaft. KK, 77. 
Baader, G., Gründer und Chefredakteur. In Idhenhausen wurde von dem Pfarrer G. Baader der Ichenhausener Volksfreund, ein Wochenblatt, im katholischen Sinne redigiert. 19, 60. - Der Volksfreund von Ichenhausen wurde gegründet von dem Pfarrer G. Baader. 18, 210.

Bach, Joseph, Mitbegründer und Publizist, geb. 4. 3. $1833 \mathrm{zu}$ Aislingen in BayrischSchwaben, gest. 24. 9. 1901 zu München, 1856 Priester, 1867 a. o. Professor und Universitätsprediger daselbst, 1872 Ordinarius für Pädagogik und Philosophie an der dortigen Theol. Fakultät, seit 1881 auch für Apologetik. Mitarbeiter der Historisch-politischen Blätter. $\mathrm{KK}, 89$. - Bach war Mitbegründer der 1855 entstandenen Zeitschrift Natur und Offenbarung. 9, 46.

Bangha, Adalbert, SJ, Publizist, geb. 19. 9. 1880 in Neutra, gest. 29. 4. 1940 in Budapest, Mitglied des 1. Internationalen Kongresses des katholischen Journalismus in Brüssel 1930. 11, 25. - Mitarbeiter der Schönere Zukunft, des Wiener Neues Reich und der Augsburger Postzeitung, wo er sich über die Situation des katholischen Pressewesens verbreitete. 11, 292-95.

Bardenhewer, Otto, Herausgeber und Mitherausgeber, geb. 16. 3. $1851 \mathrm{zu}$ MönchenGladbach, studierte in Bonn und Würzburg, promovierte zum Dr. phil. und Dr. theol., habilitierte sich 1879 in München und wurde 1884 Professor für neutestamentliche Exegese in Münster, 1886 in München. Herausgeber der Zeitschrift Biblische Studien, 1895-1916, Mitherausgeber der Bibliothek der Kirchenväter, seit 1911. KK, 106.

Barthold (?), Publizist, Mitarbeiter der Katholischen Studien. 9, 74.

Bartmann, Bernhard, Mitherausgeber, geb. 26. $5.1860 \mathrm{zu}$ Madfeld in Westf., studierte in Tübingen, wurde Lehrer an der Rektoratschule in Hamm, dann am Realgymnasium in Dortmund, und 1898 Professor der Dogmatik in Paderborn. Mitherausgeber der Zeitschrift Theologie und Glaube. KK, 109.

Batz, Johann Josef, Mitbegründer und Herausgeber, geb. 23. 6. 1775 in Bamberg, gest. 12. 3. 1814 in Bühl. 1797-1809 nacheinander Professor für Philosophie, Physik,
Mathematik und Dogmatik in Bamberg. Er vertrat die Richtung der Aufklärung, gründete die Theologische Zeitschrift, deren erste drei Bände er herausgab. (Bamberg 1809 bis 1810). L II, 53. - Batz, Prof. und Regens des Seminars in Bamberg, gab die Theologische Zeitschrift (1809-14) bis zum 4. Band heraus, 9, 19.

Baudri, Johann Anton Friedrich, Publizist und Mitbegründer, geb. 20. 2. 1804 zu Elberfeld, gest. 29. 6. 1893 zu Köln, 1843 Domkapitular, 1844 Generalvikar, 1850 Titularbischof, 1853 Domdechant, 1864-66 Erzbistumsverweser daselbst, schrieb u. a. die Biographie Kardinal Geissels 1881. KK, 112. - Mitglied der Gründungsversammlung der Rheinischen Volkshalle am 11. 4. 1848. 1 II, 8. - Mitarbeiter der Katholischen Zeitschrift für Wissenschaft und Kunst. 13, 220.

Bauer, Renward, Publizist und Mitbegründer, geb. 13. 11. 1823 zu Muri im Aargau, gest. 10. 7. $1883 \mathrm{zu}$ Kirchrath in Holland, floh 1841 ins Ausland, wurde Jesuit (Lehrer der Kirchengeschichte in den Ordenshäusern zu Bonn und Maria-Laach) und ließ sich 1873 nach der Vertreibung seines Ordens aus dem deutschen Reich in Löwen nieder. Mitbegründer und Mitarbeiter der Stimmen aus Maria-Laach. KK, 114.

Baumeister, Ansgar, Mitherausgeber, geb. 18. Juni 1873 zu Karlsruhe in Baden. Doktor der Philosophie und Theologie, Subregens und Professor am Priesterseminar in St. Peter zu Freiburg i. Br. Mitherausgeber des Oberrheinischen Pastoralblattes seit 1910. KK, 118.

Baumgarten, Friedrich, Redakteur, geb. 1816 zu Krumau im Böhmerwald, gest. 19. 5. 1882 zu Linz an der Donau. Studierte in Salzburg, wurde 1857 Stadt-Pfarrer in Wels, 1874 Domherr in Linz, 1878 StadtPfarrer in Linz, zuletzt Domdechant daselbst. Redakteur der Linzer theologischen Quartalsschrift 1850-62. KK, 119.

Baumgarten, Paul Maria, Publizist, geb. 25. 7. 1860 zu Rittershausen bei Elberfeld, seit 1894 Priester, Dr. theol. und Dr. jur. Konsistorialrat, Privatgelehrter, Memoirenschreiber und Publizist. KK, 119. - Bis 1924 in Rom mit Studien zur päpstlichen 
Diplomatie beschäftigt, verdient um die Begründung der kirchlichen Statistik. Außerdem fruchtbarer und gut unterrichteter, zuweilen indiskreter Publizist. L II, 69.

Baumgartner, Alexander, Redakteur, geb. 27. 6. 1841 zu St. Gallen, gest. 5. 9. 1910 zu Luxemburg, studierte in Münster, trat 1860 in den Jesuitenorden ein und bildete sich in Feldkirch, Maria-Laach und DittonHall aus, wurde 1867 Sprachlehrer in Feldkirch, 1870 in Stonyhust und arbeitete seither an den Stimmen aus Maria-Laach mit, deren Mitredakteur er 1877 wurde. Nach der Vertreibung der Jesuiten aus Deutschland in Luxemburg lebend, vorwiegend Literaturhistoriker und Reiseschriftsteller. KK, 120. - Mitarbeiter der Zeitschrift Katholische Missionen. 18, 53.

Bayerle, G. B., Herausgeber und Chefredakteur, Kaplan an St. Lambertus in Düsseldorf, Herausgeber und Redakteur des Rheinischen Kirchenblatt seit 21. 12. 1843. 13, 34 u. 219 . - Seit 1845 erschien die Zeitschrift Katholische Blätter in Düsseldorf, redigiert von Bayerle. 1 I, 249 und 13, 221. - Er redigierte das Rheinische Kirchenblatt bis 1848.1 I, 248.

Beck, Joseph, Chefredakteur, geb. 28. 10. $1858 \mathrm{zu}$ Sursee, Sohn des konservativen Politikers Franz Beck-Leu, 1885 Vikar zu Basel, 1888 Prof. der Exegese und Konviktsdirektor in Luzern, 1891 Professor der Pastoral in Freiburg im Uchtland, auch Sozialpolitiker. Seit 1896 Vorstandsmitglied des Schweizer Arbeiterbundes. Herausgeber der Zeitschrift für christliche Sozialreform seit 1902. KK, 133. - Mitarbeiter der Alte und neue Welt. Aut. (36. Jg. 1902, S. 534-559).

Beck, Ludwig Joseph, Publizist, geb. zu Mainz, gest. im März 1816 zu Limburg an der Lahn. Wurde Pfarrer in Kempenich, um 1780 Fiscal in Koblenz, daselbst Geheimer Rath und Generalvikar, seit 1792 in Trier. 1812 Vicarius, capituli sede vacante'. Mitarbeiter der Deutschen Blätter. A II, 217. - Mitarbeiter an den Deutschen Blättern für Protestanten und Katholiken, 1839 bis 1841 , die in Heidelberg verlegt wurden. 1 I, 239. (NB! Widersprüchliche Daten.)
Becker, Dr., Redakteur und Mitherausgeber, Gymnasial-Religionslehrer in Bonn, Mitherausgeber und Redakteur der Monatsblätter für den katholischen Religionsunterricht in höheren Schulen. KU, 746.

Becker, Joseph Blasius, Redakteur und Mitherausgeber, geb. 3. 2. 1857 in Mainz, daselbst gest. am 28. 5. 1926. 1895 Professor der Moraltheologie, 1900 für Dogmatik, 1904-20 Regens des Seminars. Seit 1907 Mitherausgeber des Katholik. L II, 92. Seit 1907 war Becker zusammen mit Professor Selb Herausgeber und Redakteur des Katholik. 1 I, 196.

Bellesheim, Alfons, Redakteur, geb. 16. 12. $1839 \mathrm{zu}$ Montjoie, gest. 5. 2. $1912 \mathrm{zu}$ Aachen, 1862 Priester, 1862-65 Kaplan an der Anima in Rom, 1865-86 Domvikar und Sekretär des Generalvikariats in Köln, seit 1886 Stiftsherr, seit 1902 Stiftspropst in Aachen und apostolischer Protonotar. Kirchenhistoriker. KK, 150. - Bellesheim wurde Mitarbeiter der Kölnischen Volkszeitung und redigierte die Auslandspolitik unter besonderer Berücksichtigung kirchlicher Fragen. 1 III, 108.

Benkert, Franz Georg, Herausgeber, geb. 25. 9. $1970 \mathrm{zu}$ Nordheim an der Rhön, gest. 26. 5. 1859 zu Koburg, 1821 Subregens, 1832 Regens im Priesterseminar, seit 1838 Domdechant in Würzburg, gab seit 1822 den Religionsfreund für Katholiken und die Zeitschrift für Pastoraltheologie Athanasia heraus, verdient um die Wiedergeburt des kirchlichen Geistes im katholischen Deutschland. $\mathrm{KK}, 154 .-1822-47$ Herausgeber und 1822-36 Redakteur des Religionsfreund für Katholiken, ab 1828 umbenannt in Allgemeiner Religions- und Kirchenfreund, in Würzburg. 13, 200 vgl. 1 I, 245 und 9, 22.

Berg, Philipp von, Publizist, 1815 in Köln geboren, studierte in Bonn und Breslau, dazu in Rom Theologie, 1843 zum Priester geweiht, danach Kaplan in Jülich und in Rheindorf bei Bonn. 1848 Abgeordneter in Frankfurt. A II, 364. - Mitarbeiter am Nathanael. 13, 107. 